\title{
Surgical Innovation in the Era of Global Surgery
}

\author{
A Network Analysis \\ George Garas, MD, FRCS, ${ }^{*} \dagger$ Isabella Cingolani, PhD, $\ddagger$ Vanash Patel, MD, PhD, FRCS, ${ }^{*}$ \\ Pietro Panzarasa, PhD, § Derek Alderson, MD, FRCS, † \\ Ara Darzi, MD, KBE, FRCS, ${ }^{*} \dagger$ and Thanos Athanasiou, MD, PhD, MBA, FRCS ${ }^{*} \dagger$
}

\begin{abstract}
Objective: To present a novel network-based framework for the study of collaboration in surgery and demonstrate how this can be used in practice to help build and nurture collaborations that foster innovation.

Background: Surgical innovation is a social process that originates from complex interactions among diverse participants. This has led to the emergence of numerous surgical collaboration networks. What is still needed is a rigorous investigation of these networks and of the relative benefits of various collaboration structures for research and innovation.

Methods: Network analysis of the real-world innovation network in robotic surgery. Hierarchical mixed-effect models were estimated to assess associations between network measures, research impact and innovation, controlling for the geographical diversity of collaborators, institutional categories, and whether collaborators belonged to industry or academia.

Results: The network comprised of 1700 organizations and 6000 links. The ability to reach many others along few steps in the network (closeness centrality), forging a geographically diverse international profile (network entropy), and collaboration with industry were all shown to be positively associated with research impact and innovation. Closed structures (clustering coefficient), in which collaborators also collaborate with each other, were found to have a negative association with innovation $(P<0.05$ for all associations). Conclusions: In the era of global surgery and increasing complexity of surgical innovation, this study highlights the importance of establishing open networks spanning geographical boundaries. Network analysis offers a valuable framework for assisting surgeons in their efforts to forge and sustain collaborations with the highest potential of maximizing innovation and patient care.
\end{abstract}

Keywords: collaboration, innovation, network analysis, research, surgery

(Ann Surg 2018;xx:xxx-xxx)

From the *Surgical Innovation Center, Department of Surgery and Cancer, Imperial College London, St. Mary's Hospital, London, UK; †Department of Surgical Research and Innovation, The Royal College of Surgeons of England, London, UK; $\ddagger$ Big Data and Analytical Unit, Imperial College London, St. Mary's Hospital, London, UK; §School of Business and Management, Queen Mary University of London, London, UK; and 9 Department of Surgery, University of Birmingham, Queen Elizabeth Hospital, Birmingham, UK.

Dr. George Garas, MD, FRCS, holds a Royal College of Surgeons of England Doctoral Research Fellowship (Grant number GG 1037600/2017-2018) and is also supported by Imperial College London (Grant number CID 337755/20152018 ) and the Alexander S. Onassis Public Benefit Foundation (Grant number F ZM 014-1/2016-2017). The funders had no role in the study design, data collection and analysis, decision to publish, or preparation of the manuscript.

The authors report no conflicts of interest.

Supplemental digital content is available for this article. Direct URL citations appear in the printed text and are provided in the HTML and PDF versions of this article on the journal's Web site (www.annalsofsurgery.com).

Reprints: George Garas, MD, FRCS, Specialty Registrar and Honorary Clinical Lecturer in Surgical Oncology and Endocrine Surgery, Department of Surgery and Cancer, Imperial College London, 10th Floor, QEQM Wing, St. Mary's Hospital, London W2 1NY, UK. E-mail: g.garas@imperial.ac.uk.

Copyright (C) 2018 Wolters Kluwer Health, Inc. All rights reserved.

ISSN: 0003-4932/16/XXXX-0001

DOI: $10.1097 /$ SLA.0000000000003164
A $\mathrm{s}$ a result of recent technological and computational advances and the exponential rate at which new knowledge is generated, the "lone innovator" model no longer represents a suitable paradigm for scientific production. ${ }^{1}$ In the modern world, innovation is increasingly the outcome of a collaborative process. ${ }^{2}$ This is especially the case for surgical innovation that has been unfolding at a global scale, within a complex network of international collaborations. $^{3-5}$

The aim of collaborative efforts is the generation of social capital. The definition and generative mechanisms of social capital have long been the subject of debates and controversies within the social sciences. ${ }^{6,7}$ Typically, scholars tend to converge on the idea that social capital refers to the value that individuals, groups, or organizations can derive from the underlying social relations. ${ }^{8}$ In the context of surgical research, social capital can relate to accessing data, expertise, knowledge, or any other type of resources that become available through specific collaborative patterns and facilitate knowledge creation and innovation.

Surgical innovation networks are complex systems, typically consisting of hundreds or thousands of organizations dispersed across the globe forging various types of relationships with one another. ${ }^{5}$ Network science offers a theoretical and methodological backdrop that has recently been widely used to study collaboration and innovation in a variety of fields. However, a network approach to examining surgical innovation has been largely neglected. ${ }^{9,10}$

This study applied network analysis to a real-world global collaboration network in robotic surgery. The aim was to familiarize surgeons with network analysis and demonstrate how this approach can be used to devise effective strategies toward the establishment of partnerships that can enhance research impact, facilitate innovation, and advance patient care.

\section{METHODS}

\section{The Dataset}

This study draws on the Web of Science (WOS) platform (Clarivate Analytics, Philadelphia, PA). All articles on robotic surgery were extracted through the use of the MeSH terms: "robot $O R$ robotic $O R$ robot assisted OR robotic assisted OR robotically assisted OR robot-assisted OR robotic-assisted OR roboticallyassisted." The Research Area was confined to "Surgery" and the Document Types to "Article."

The search was performed on January 17, 2017 and produced 3889 publications (peer-reviewed articles) published between July 1988 and January 2017 (Figure S1, http://links.lww.com/ SLA/B554). These were all used for constructing the coauthorship network. Articles were generated from 1700 organizations nested within 62 countries, in turn nested within 6 geographical regions (Table S1, http://links.lww.com/SLA/B554). 


\section{The Surgical Collaboration Network}

The collaboration network was constructed using VOSviewer (Leiden University, Leiden, The Netherlands), a software developed specifically for the study of scientific collaboration networks. In the network, each node represents an organization, and a link between 2 nodes represents collaboration between the corresponding organizations. As coauthorship has been shown to be a good proxy of collaboration, ${ }^{11,12}$ links between nodes were based on coauthorship of articles. The resulting network is weighed: the value (or weight) of a collaborative link increases as a function of the intensity of collaboration (Figure S2, http://links.lww.com/SLA/B554).

\section{Outcome Measures}

For each organization, 2 outcome measures were computed: 1 ) the research impact; and 2) the innovation index. These measures were computed as follows:

Research impact: Research impact was measured as the sum of normalized citations received by all articles (co-) authored by scholars affiliated with a given organization in each year. To obtain normalized citations, the citation count for each publication in a given year was divided by the average number of citations obtained by all articles published in the same year. The greater the sum of normalized citations for a given organization, the greater the organization's research impact (ie, normalized citations are used to measure impact).

- Innovation index: The innovation index represents a recently validated metric used to evaluate and rank surgical innovation (Table S2, http://links.lww.com/SLA/B554). ${ }^{13}$ It captures the value of the innovative output produced by an organization as a function of the degree to which it reached an implementation stage (Figures S3-S4, http://links.lww.com/SLA/B554). Thus, the greater the innovation index of a given organization, the more innovative the organization's surgical research output.

For a detailed description of how each performance metric was calculated, please see sections S.3.1 and S.3.2 in the Supplementary Material, http://links.lww.com/SLA/B554.

\section{Network Measures}

Two established network measures were computed: 1) the clustering coefficient; and 2) closeness centrality. These measures were defined as follows:

- Local clustering coefficient: The local clustering coefficient quantifies how closed an organization's ego-centered network is (ie, the network including connections between the organization and its partners as well as connections between these partners) enabling assessment of the extent to which an organization's collaborators also collaborate with each other or, alternatively, the extent to which an organization spans structural holes separating collaborators. The higher the local clustering coefficient of an organization, the more closed the organization's ego-centered network is. More specifically, the local clustering coefficient of an organization was defined as the ratio between the number of actual triangles containing the organization and its neighbors, and the maximum possible number of such triangles. A generalized weighted clustering coefficient was calculated to take into account the weights of links (see Supplementary Material, http://links.lww.com/SLA/B554). To facilitate interpretation, all values of the generalized weighted clustering coefficient were standardized. ${ }^{14}$ Measuring the density of triangles in an organization's local network uncovers how open or closed the network is, and the extent to which the organization acts as the knowledge broker between otherwise disconnected organizations in the collaboration network.
- Closeness centrality: The closeness centrality of an organization measures how close the organization is to all other organizations in the collaboration network. The higher an organization's closeness centrality, the greater the organization's access to the knowledge (or data, or any other resource) provided by other organizations in the collaboration network, and thus the greater the organization's influence on others as a result of its structural position. ${ }^{15}$ The generalized weighted version of closeness centrality was used to account for the weights of links (see Supplementary Material, http://links.lww.com/SLA/B554). ${ }^{16}$

For a detailed description of how each network metric was calculated, please see sections S.3.3 and S.3.4 in the Supplementary Material, http://links.lww.com/SLA/B554.

\section{Measuring the Geographical Dispersion of Collaborations}

For each organization, the geographical entropy of collaborations was computed to capture the geographical dispersion of the organization's collaborators. An organization's geographical entropy increases as the organization collaborates with other organizations located in more countries and devotes an equal amount of collaborative effort towards each of these countries (Figure S5, http://links.lww.com/SLA/B554). For a detailed description of how geographical entropy was measured, please see section S.3.5 in the Supplementary Material, http://links.lww.com/SLA/B554.

\section{Measuring Academic-industry Collaborations}

All organizations that were publicly registered as companies and classified as "corporate" entities in the WOS platform (through the InCites intelligence tool) were identified. ${ }^{17,18}$ For each organization, the sum of the organization's collaborative efforts toward other industrial (corporate) partners during the study period was calculated. For a detailed description of how the strength of industrial collaborations was measured, please see section S.3.6 in the Supplementary Material, http://links.lww.com/SLA/B554.

\section{Control Variables}

Many other organizational characteristics may influence scientific performance. This study controlled for the following 2 additional variables: 1) each organization's institutional type (eg, academic, corporate, health, etc. as classified by the InCites intelligence tool in the WOS platform) and 2) a measure of volume, here referred to as "number of articles in WOS," given by the number of all articles, beyond robotic surgery, published by each organization that the InCites intelligence tool could retrieve in the WOS database (see section S.3.7 in Supplementary Material, http://links.lww.com/SLA/B554). Controlling for research volume enables the association between collaboration network and both citation count and innovation to be investigated by keeping the number of publications constant.

\section{Statistical Analysis}

Maximum-likelihood estimates of 2 hierarchical 3-level random-intercept models were computed in which organizations were nested within countries, in turn nested within geographical regions. A random-intercept structure was combined with heteroskedastic level1 residuals by letting the variances of these residuals be a function of the organization's institutional type. All models were estimated using STATA 15 (StataCorp LP, College Station, TX). The significance threshold was set at $P<0.05$.

\section{RESULTS}

Of the 1700 organizations, 1543 were connected through at least 1 collaboration. The overall network comprised of $6000 \mathrm{col}-$ laborative links. Figure 1 shows 2 extreme examples of ego-centered 


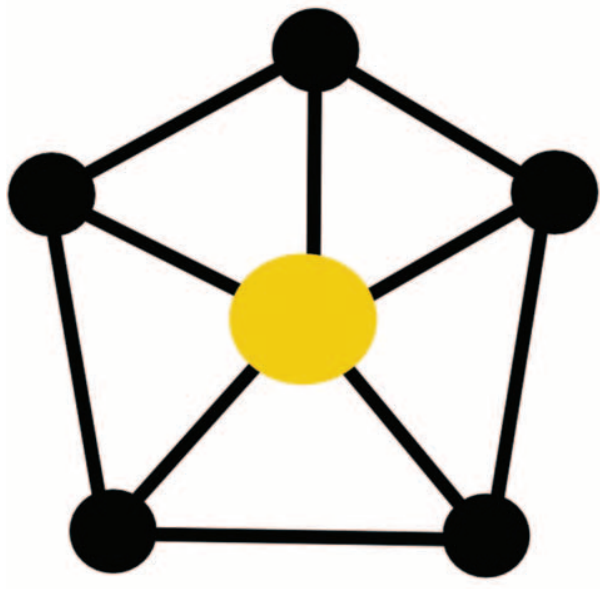

A Closed structure

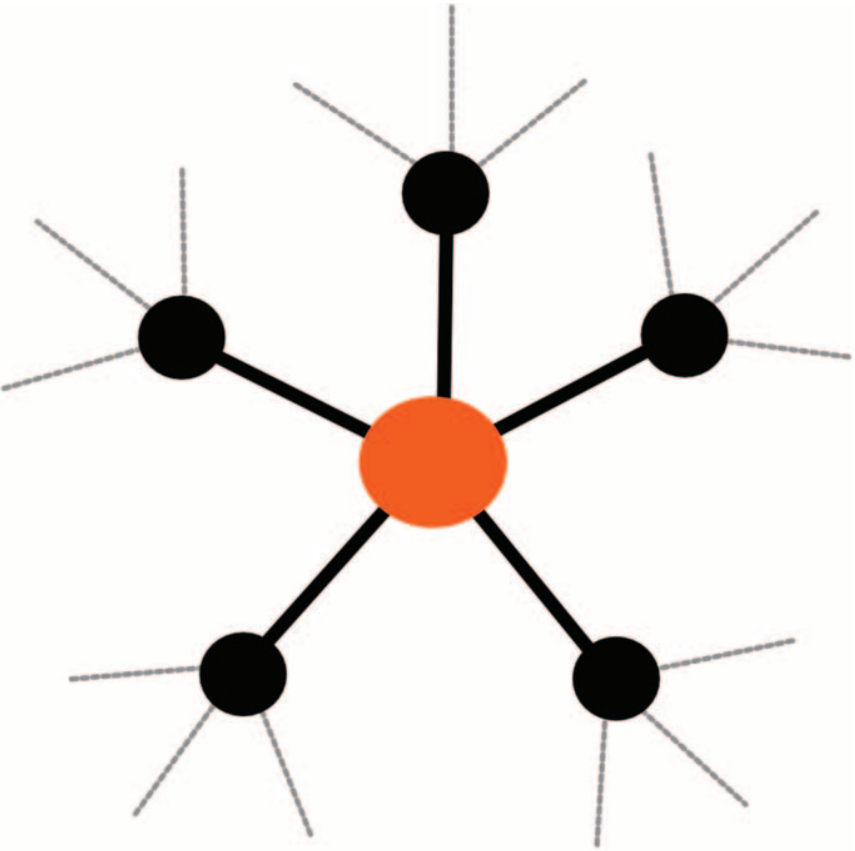

B
Open structure

FIGURE 1. Illustrative examples of the 2 extreme cases of a closed ego-centered network (A) and an open ego-centered network (B). The closed network is rich in third-party relationships and closed triangles: all nodes connected to ego (yellow node) are also connected with each other. The open network is rich in brokerage opportunities and open triads: ego (orange node) acts as the broker between all contacts that would otherwise be unable to reach one another. Ego in the closed network has therefore a clustering coefficient equal to one, while ego in the open network a clustering coefficient equal to zero.

network, 1 characterized by a closed structure $\left(c c_{\text {org }}=1\right)$ rich in third-party relationships (Fig. 1A), and the other by an open structure $\left(c c_{\text {org }}=0\right)$, rich in brokerage opportunities (Fig. 1B).

The collaboration network among organizations is shown in Figure 2. While the highest-performing organizations (eg, University of Pittsburgh and Yonsei University) achieved the largest research impact (node size) and innovation value (node color), Figure $2 \mathrm{~A}$ suggests that the correlation between the 2 performance measures is far from perfect. Successful organizations (Fig. 2C) appear to be better connected than less successful ones (Fig. 2B), which are more sparsely connected.

Table 1 shows the maximum-likelihood estimates of the coefficients and standard errors of the 2 hierarchical random-intercept models of research impact and innovation. The first 2 estimated parameters in both models suggest that both citations and innovation value at the organizational level were statistically significantly associated with the organization's position in the collaboration network. The local clustering coefficient at the organizational level was negatively associated with both performance measures, although only the association between clustering and innovation reached statistical significance. Both geographical entropy and industrial collaboration were positively and statistically significantly associated with both research impact and innovation. Estimates for all remaining fixed-effect and random-effect parameters are shown in Tables S3, http://links.lww.com/SLA/B554 and S5, http://links.lww.com/SLA/B554 (see also Tables S4, http://links.lww.com/SLA/B554 and S6-S8, http:// links.lww.com/SLA/B554 for robustness checks).

Figure 3 shows the topology and properties of 4 ego-centered networks of selected organizations that differed in terms of both innovation index and clustering coefficient. For instance, Figure 3A suggests that Leiden University was characterized by a closed egocentered network in which the collaborators tended to collaborate with one another, while Imperial College London (Fig. 3C) was positioned in a more open network, rich in structural holes, and opportunities for brokerage between collaborators. In turn, Imperial College London was associated with a higher innovation index than Leiden University, which indicates that organizations can extract value from the structural cleavages separating their partners.

Figure 4 shows the association between closure of ego-centered networks (node size) and both measures of performance (node color). Figure 4A does not suggest an unambiguous relationship between network closure and research impact, as both large and small nodes can be associated with high performance. Figure 4B, however, indicates that nodes within closer structures were associated with lower values of innovation index. Organizations that produced more innovative outcomes were those that spanned structural holes between collaborators.

\section{DISCUSSION}

This study was concerned with social capital in surgical research, and has uncovered structural sources of research impact and innovation. The study examined the relative benefits of 2 opposing structures-closed and open networks-by analyzing the ego-centered networks of all organizations that published research on robotic surgery between 1988 and 2017. We investigated the association between local clustering coefficient and a 2-fold measure of research performance: impact and implementation-based innovation value. Closed structures, rich in third-party relationships, were 

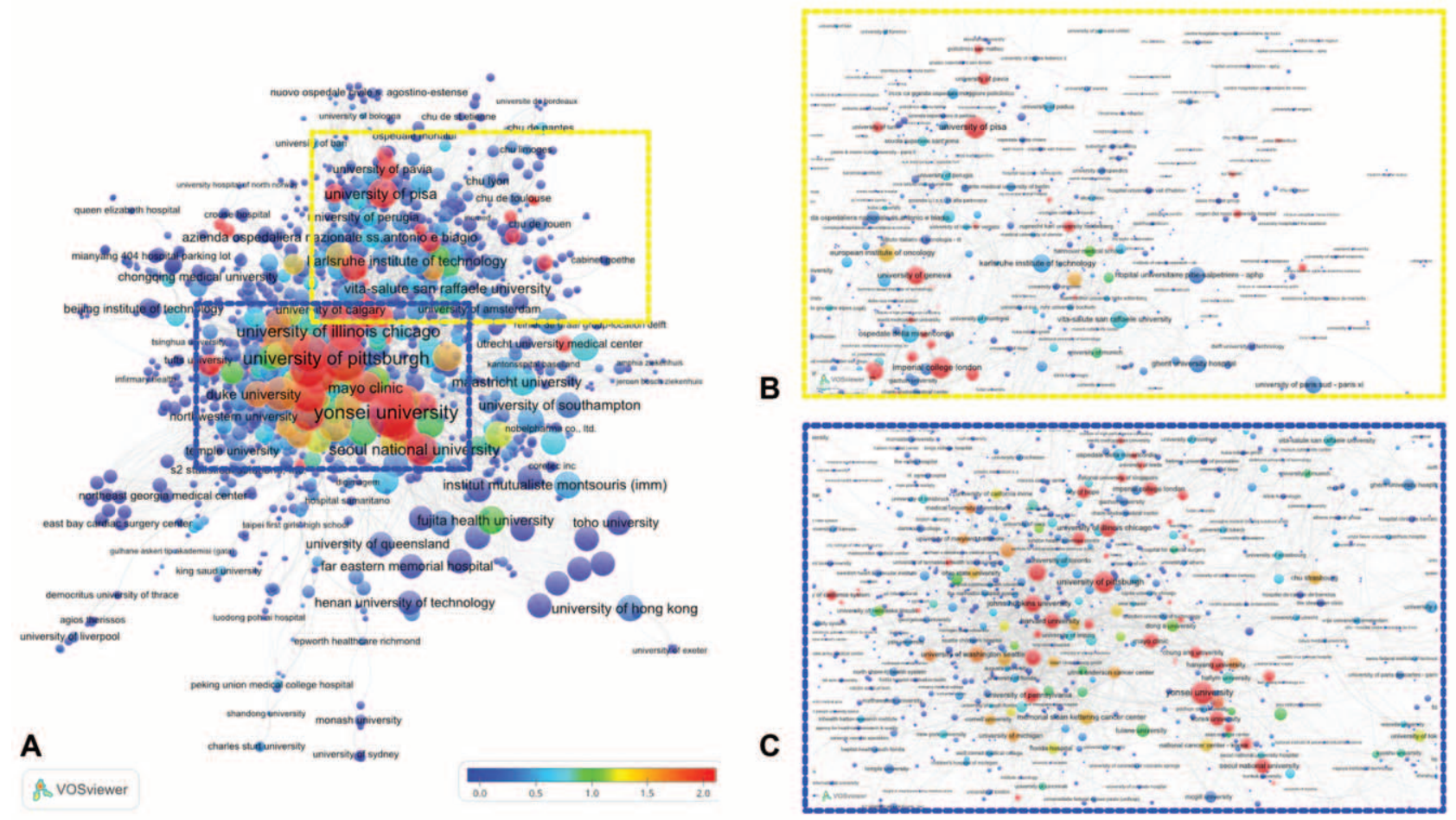

FIGURE 2. The global robotic surgery collaboration network. The size of each node is proportional to the average normalized citations of the corresponding organization, while the color is proportional to the innovation index. The weight of each link (ie, the thickness of the line connecting any 2 nodes) is proportional to the normalized count of collaborations between the connected pair of organizations. Panel A shows the largest connected component of the collaboration network. Panel B shows a subset of less successful organizations, more peripheral, and poorly connected. Panel C shows a subset of more successful organizations, highly connected and centrally located within the global network.

negatively associated with both measures, although only the association with the latter was statistically significant.

It is difficult to identify the reason(s) underlying these findings. A plausible explanation (though this was not tested and would form the subject of a separate study) is that nodes in a closed structure are more likely to cite each other, thus inflating their research impact (citations), which would explain the lack of statistical significance of the negative association between clustering and research impact. At the same time, a closed structure would naturally limit the opportunities for brokerage between collaborators and in so doing compromise their ability to translate novel ideas, products, or surgical procedures they may have developed and published into truly innovative outcomes that could subsequently be implemented on a large scale. This would explain the negative and statistically significant association between closed structures (clustering) and innovation.

Closed networks may still be important to surgical innovation. They may facilitate distributed understanding, distributed ownership, and the application of complex ideas. ${ }^{7}$ However, the present findings suggest that open networks and brokerage opportunities are vital for fostering truly innovative outcomes. Open networks enhance innovation by providing the necessary conditions and opportunities for novel combinations or rearrangements of ideas, technologies, processes and for transforming them into well-established, widely

TABLE 1. Maximum-likelihood Estimates From Three-level Random-intercept Linear Models of Research Impact (Measured by the Sum of Normalized Citations, Snc) and Innovation Value (ii)

\begin{tabular}{lcc}
\hline & Research Impact (snc) & Innovation Value (ii) \\
\hline Clustering coefficient $(c c)$ & $-0.4027(0.6165)$ & $\mathbf{0 . 0 8 2 0}(0.0284)$ \\
Closeness centrality $(l)$ & $\mathbf{6 . 0 7 8 2}(0.7253)$ & $\mathbf{0 . 2 1 6 6}(0.0332)$ \\
Geographical entropy $(\varepsilon)$ & $\mathbf{1 0 . 5 5 9 9}(0.7564)$ & $\mathbf{0 . 1 6 2 9}(0.0368)$ \\
Industrial collaboration $(i c)$ & $\mathbf{5 . 2 1 0 4}(\mathbf{1 . 2 8 3 7})$ & $\mathbf{0 . 1 9 6 7}(0.0686)$ \\
Number of articles in WOS $(v)$ & $0.0015(0.0022)$ & $0.0001(0.0001)$ \\
Number of observations* & 639 & 639
\end{tabular}

Below each estimated parameter, the corresponding standard error is reported within brackets. estimated parameters that are statistically significant at the $5 \%$ level are shown in bold.

${ }^{*}$ The regression models were estimated based on a sample of 639 organizations as these were the observation units with nonmissing values across all the covariates used (see Supplementary Material for details, http://links.lww.com/SLA/B554). 

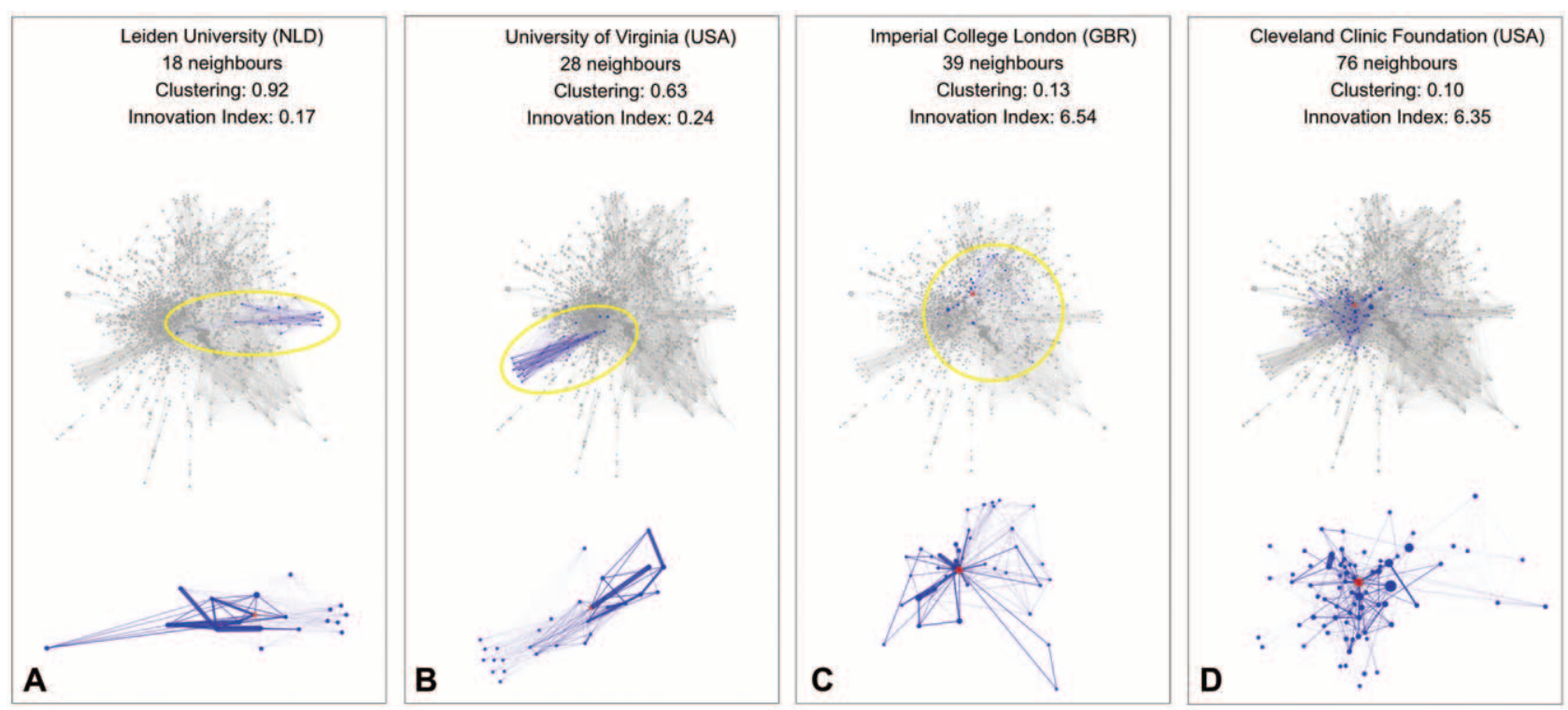

FIGURE 3. Ego-networks of 4 selected organizations, with decreasing values of clustering coefficient and increasing values of innovation index. In each panel, the ego-centered networks are identified by the yellow circles (above), and zoomed out (below).

implemented products, medical devices, or surgical procedures. This is achieved through a variety of mechanisms including access to external knowledge, data, infrastructure, and/or expertise not available "in-house," and the sharing of otherwise prohibitive costs and risks that represent 2 of the greatest barriers to innovation. ${ }^{9}$ These mechanisms are particularly salient to modern surgical research which is expensive, highly regulated, and increasingly reliant on the diverse inputs from individuals across a variety of backgrounds (including scholars not directly related to surgery such as engineers, computer scientists, and statisticians). ${ }^{19}$

This study has shown a positive and statistically significant association between closeness centrality and organizational performance. This has important implications for strategy and policy as it can assist both academic surgeons and policy-makers in their selection of organizations that have better access to others. ${ }^{15}$ For instance, focusing on closeness centrality can prove crucial in a number of
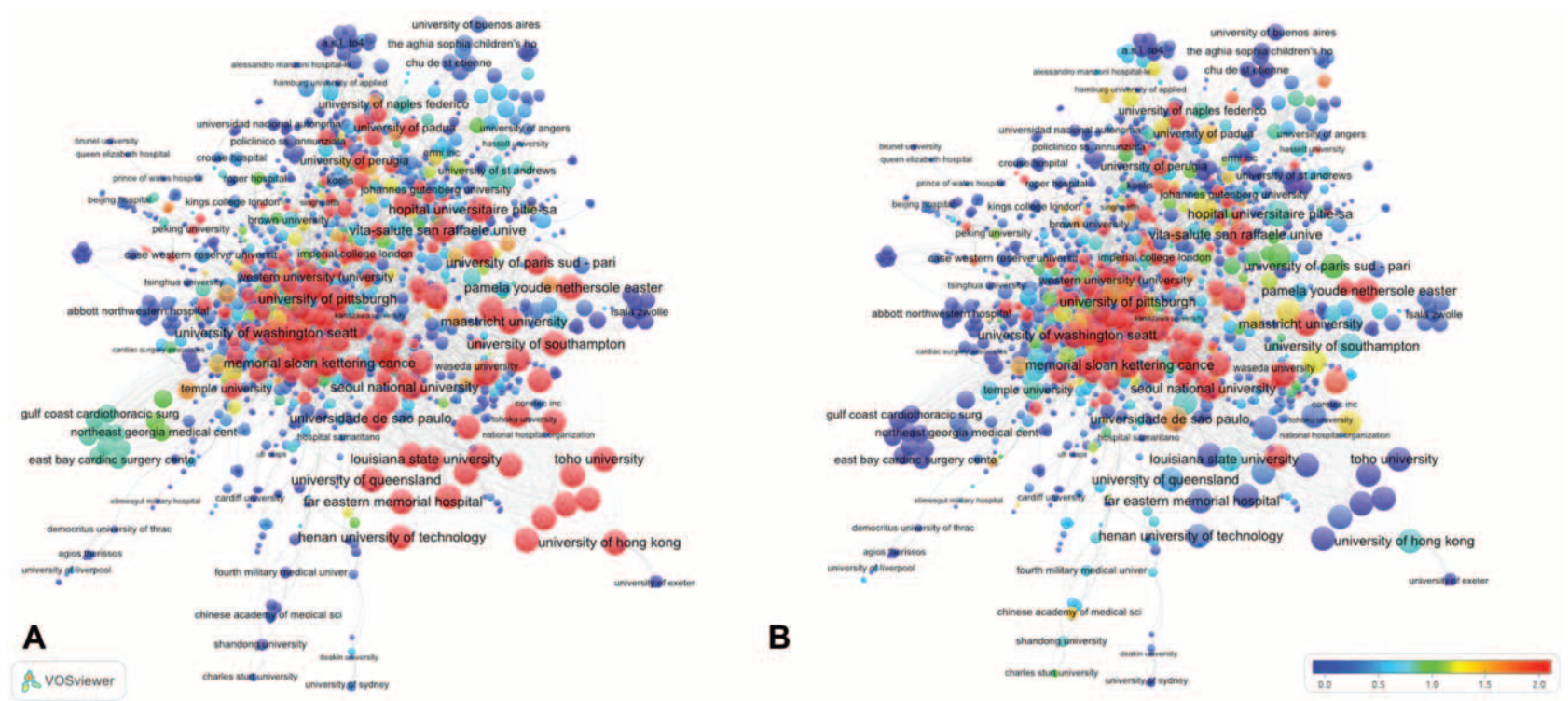

FIGURE 4. The association between closure of ego-centered networks (ie, size of nodes) and both measures of performance (ie, color of nodes). In (A), the color is proportional to research impact, while in (B) to innovation index. In both panels, the size of each node is proportional to the number of closed triangles including the node. While there is no clear-cut relationship between network closure and research impact (ie, there are both large and small red nodes in (A)), nodes in closer structures are associated with lower values of innovation index (ie, most large nodes tend to be the blue ones in (B)). 
scenarios in surgical research such as the optimal setup of multicenter randomized controlled trials where recruitment can be challenging and a large sample size is needed. ${ }^{20}$

Our findings have also highlighted the importance of maximizing an organization's geographical dispersion in terms of the location of research partners. Geographical entropy represents a complementary, yet distinct, dimension to structural openness. It reinforces the positive association between brokerage and performance by shedding light on the benefits that an organization can extract from a (geographically) diverse collaboration network (see Supplementary Material, http://links.lww.com/SLA/B554).

It is encouraging to see that global surgery has been recently gaining increasing attention through a number of collaborative initiatives. ${ }^{3,4}$ While these initiatives have been shown to provide well-known benefits to surgical education and global health (through the specialized service, training, and equipment provision to the developing world), ${ }^{3,4}$ this study suggests that they can also enhance the research impact and innovative output of all partners involved. Thus, this study's findings have important implications as they can inform policy-makers in their efforts to devise effective collaborative international strategies and surgical research policies which should aim to incentivize organizations to collaborate more globally, also with partners in the developing world.

This study has demonstrated a positive association between academic-industrial partnerships on the one hand, and research impact and surgical innovation on the other. In the corporate sector, such partnerships are actively sought, as they are known to maximize profits. From the perspective of academia, however, there is more ambiguity and controversy on their advantages. ${ }^{21}$ On average, industry contributes less than $10 \%$ of funding for academic research, and joint academic-industrial partnerships contribute to only a small fraction of the overall knowledge generated. ${ }^{17}$ Many possible reasons for this have been put forward. The most widely accepted one relates to corporate pressures diverting academic researchers away from their scientific efforts, toward commercialization. ${ }^{17}$

The positive association between collaboration with industry and research performance identified in this study may be rooted in key differences between innovation in surgery and in other fields. Surgical research and the introduction of new technology (such as robotic surgery) can be expensive. ${ }^{20}$ This, combined with the fact that governmental funding for healthcare research allocated to surgery tends to be not higher than 5\% (3\% in the USA and 5\% in the UK), engenders the need for seeking alternative sources of funding. ${ }^{22,23}$

The medical device industry, with its continuing steady growth in revenues (projected to reach $\$ 398$ billions by 2023) amid periods of economic downturns across the Western world, represents an ideal collaboration partner in some respects. ${ }^{24}$ Not only does industry possess the scale of funds that surgical research often needs (as well as other vital resources including laboratories and human capital) but it also has a strong interest in investing in surgical research (through intellectual property acquisition and subsequent commercialization of innovations). ${ }^{24}$

The internal organizational mechanisms of corporations make them highly experienced in managing various types of resources. This is likely to result in a more efficient division of labor, ${ }^{17}$ and higher research productivity of academic surgeons, typically constrained by demanding clinical and educational commitments. Through the provision of support staff to assist with time-consuming, bureaucratic activities (such as the drafting of applications for ethical committee approval, the performance of standardized laboratory work, and patient recruitment and follow-up), industrial collaboration can further free academic surgeons to concentrate on their research. ${ }^{17}$ It is encouraging to see that world-leading universities have already recognized the strategic salience of collaborations with industrial partners, and have set up dedicated liaison offices and even innovation hubs to act as "incubators" for innovation. ${ }^{25,26}$ Academic institutions involved in surgical innovation should be encouraged to follow their example.

Despite those encouraging findings, it is important to recognize that industry partnerships may not always be beneficial. Caution should be exerted, especially when corporate research funding forms part of the partnership because this will inevitably promote conflicts of interest (including those concerned with intellectual property and ownership of the innovations $)^{27}$ as the goals of industry and academia do not often align - the former is predominantly driven by commercial incentives while the latter by serving the public good. ${ }^{28}$ Problems can range from subliminal biases (eg, surgeons opting for expensive medical devices in the absence of evidence on superiority to existing ones) all the way to research misconduct (eg, companies suppressing the publication of trial results not favoring their sponsored products) generating ethical concerns. ${ }^{29}$ It is however reassuring that the severity of these problems is now widely recognized and a number of measures have been taken to counteract them. Examples include the establishment of the Physician Payments Sunshine Act and initiatives from the International Committee of Medical Journals Editors regarding clinical research governance that include the compulsory registration of all clinical trials and submission of conflicts of interest disclosure forms by all authors. ${ }^{30}$

The present study has a number of strengths and weaknesses. Its main strength lies in the novel network-based perspective on surgical research. Network science provides a comprehensive array of theories and methods for mapping and understanding collaboration patterns. ${ }^{31}$ Despite its extensive use across the social sciences and innovation studies, ${ }^{1}$ its application to the study of collaboration patterns in healthcare has so far remained relatively limited with only a handful of published studies..$^{11,15,32,33}$ In particular, most studies on surgical collaboration did not rely upon network metrics beyond the mere number of participating organizations and their corresponding countries. ${ }^{3,5,34-37}$ We took a step in this direction by computing both local (ie, clustering coefficient) and global (ie, closeness centrality) measures that account not only for each organization's connections with collaborators but also for connections these collaborators have with one another (in the organization's ego-centered network) and with other organizations (in the global network).

This study also engaged with current debates and controversies on social capital and its structural sources. The fundamental role of networking in surgical research for both innovation and patient care is widely recognized, ${ }^{5}$ and an increasing number of national and global surgery initiatives have been recently established..$^{3,34-37}$ However, which type of networking pattern (eg, open vs closed structures) matters has remained largely unexplored. The present study investigated the relative benefits of different collaboration patterns for both research impact and surgical innovation. The findings have suggested that both collaborative brokerage and geographical boundary spanning are catalysts of surgical innovation. Much of the network and innovation literature has failed to distinguish between these 2 mechanisms, although they remain conceptually and empirically distinct. This study contributed to disentangle them by suggesting that surgical innovation can be further enhanced when boundary-spanning leaders leverage collaborative brokerage, and brokering leaders amplify the spatial diversity of their collaborative network.

Limitations included the fact that the collaboration network was constructed based on coauthorship, and therefore did not reflect any other form of informal intellectual exchange (eg, mentorship, discussion, informal commentary) that did not result in a publication. Fully accounting for the problem of the opaqueness of collaboration would inevitably be an arduous task, especially when conducted on a 
large scale, and we believe our results still remain fairly robust against possible biases that using coauthorship as proxy for collaboration might induce. ${ }^{11,12}$ Another limitation of the current analysis lies in its cross-sectional nature. Future work might consider investigating the evolution of the collaboration network over time and uncovering the dynamics of social capital. Collaboration was only evaluated at the meso (organizational) level. It will be interesting for future studies to evaluate structural sources of innovation and research impact at the micro (individual) and macro (country) levels as well as sources of other innovations in surgery (eg, augmented reality for intraoperative navigation in robotic surgery and 3-dimensional printing).

This study can be regarded as a proof of concept suggesting how network analysis can be used in surgical research to foster innovation and thus patient care through strategic partnerships. The findings, showing that innovation is inherently a social process, have a number of implications that can potentially inform policy-makers and funding bodies. Evidence was provided on the ways in which existing collaborative efforts can be adjusted and future ones strategically planned to maximize research performance.

\section{REFERENCES}

1. Arena M, Cross R, Sims J, et al. How to catalyze innovation in your organization. MIT Sloan Manage Rev. 2017;58:39-47.

2. Davis JP, Eisenhardt KM. Rotating leadership and collaborative innovation: recombination processes in symbiotic relationships. Administrative Sci Quart 2011;56:159-201.

3. Chao TE, Riesel JN, Anderson GA, et al. Building a global surgery initiative through evaluation, collaboration, and training: the Massachusetts General Hospital experience. J Surg Educ. 2015;72:e21-e28.

4. Davies JI, Meara JG. Global surgery—going beyond the Lancet Commission. Lancet. 2015;386:507-509.

5. Soreide K, Alderson D, Bergenfelz A, et al. Strategies to improve clinical research in surgery through international collaboration. Lancet. 2013;382:1140-1151.

6. Latora V, Nicosia V, Panzarasa P. Social cohesion, structural holes, and a tale of two measures. J Stat Phys. 2013;151:745-764.

7. Fleming L, Mingo S, Chen D. Collaborative brokerage, generative creativity, and creative success. Administrative Sci Quart. 2007;52:443-475.

8. Social Capital Research. Definition of Social Capital. 2017; Available at: https://www.socialcapitalresearch.com/literature/definition.html. Accessed December 18, 2018

9. Ye J, Kankanhalli A. Exploring innovation through open networks: a review and initial research questions. IIMB Manage Rev. 2013;25:69-82.

10. Garas G, Cingolani I, Panzarasa P, et al. Beyond IDEAL: the importance of surgical innovation metrics. Lancet. 2018. In press.

11. Ortega JL, Aguillo IF. Institutional and coutry collaboration in an online service of scientific profiles: Google Scholar Citations. J Informetrics. 2013;7:394-403.

12. Smith M. The trend toward multiple authorship in psychology. Am Psychol. 1958;13:596-599.

13. Garas G, Cingolani I, Panzarasa P, et al. Network analysis of surgical innovation: measuring value and the virality of diffusion in robotic surgery. PLoS One. 2017;12:e0183332.
14. Opsahl T, Panzarasa P. Clustering in weighed networks. Social Networks. 2009;31:155-163.

15. Su Y, Long C, Yu Q, et al. Global scientific collaboration in COPD research. Int J Chron Obstruct Pulmon Dis. 2017;12:215-225.

16. Opsahl T, Agneessens F, Skvoretz J. Node centrality in weighed networks: generalizing degree and shortest paths. Social Networks. 2010;32:245-251.

17. Savage N. Industry links boost research output. Nature. 2017;552:S11-S13.

18. Web of Science ${ }^{\mathbb{R}}$. InCites Indicators Handbook. Clarivate Analytics. 2018; Philadelphia, PA.

19. O'Sullivan GC. Advancing surgical research in a sea of complexity. Ann Surg. 2010;252:711-714.

20. Garas G, Ibrahim A, Ashrafian H, et al. Evidence-based surgery: barriers, solutions, and the role of evidence synthesis. World J Surg. 2012;36: 1723-1731.

21. Bjerregaard T. Universities-industry collaboration strategies: a micro-level perspective. Eur J Innovation Manage. 2009;12:161-176.

22. Royal College of Surgeons of England. Funding Surgical Research. 2017 London, UK. Available at: https://www.rcseng.ac.uk/about-the-rcs/supportour-work/funding-surgical-research/. Accessed December 18, 2018

23. Mann M, Tendulkar A, Birger N, et al. National institutes of health funding for surgical research. Ann Surg. 2008;247:217-221.

24. Wong KA, Hodgson L, Garas G, et al. How can cardiothoracic and vascular medical devices stay in the market? Interact Cardiovasc Thorac Surg. 2016;23:940-948.

25. Imperial College London Faculty of Medicine. Corporate Pantnerships. 2018; London, UK. Available at: http://www.imperial.ac.uk/medicine/partnership/ corporate-partnerships/. Accessed December 18, 2018

26. Johns Hopkins School of Medicine. Industrial Liaison Office. 2018; Baltimore, MD. Available at: https://www.bme.jhu.edu/about/careers/industrialliaison/. Accessed December 18, 2018

27. Behrens TR, Gray DO. Unintended consequences of cooperative research: impact of industry sponsorship on climate for academic freedom and other graduate student outcome. Res Policy. 2001;30:179-199.

28. Welsh R, Glenna L, Lacy W, et al. Close enough but not too far: assessing the effects of university-industry research relationships and the rise of academic capitalism. Res Policy. 2008;37:1854-1864.

29. Baird P. Getting it right: industry sponsorship and medical research. CMAJ. 2003; $168: 1267-1269$.

30. Camilleri M, Tremaine WJ. Governance of clinical research. Am J Gastroenterol. 2012;107:336-338.

31. Newman MEJ. Networks. An Introduction.. Oxford, UK: Oxford University Press; 2018.

32. Chen K, Yao Q, Sun J, et al. International publication trends and collaboration performance of China in healthcare science and services research. Isr J Health Policy Res. 2016;5:1.

33. Gonzalez-Alcaide G, Park J, Huamani C, et al. Evolution of cooperation patterns in psoriasis research: co-authorship network analysis of papers in medline (1942-2013). PLoS One. 2015;10:e0144837.

34. Campbell B, Patrick H, REGISTER Group. International collaboration in the use of registries for new devices and procedures. Br J Surg. 2012;99:744-745.

35. Jamjoom AA, Phan PN, Hutchinson PJ, et al. Surgical trainee research collaboratives in the UK: an observational study of research activity and publication productivity. BMJ Open. 2016;6:e010374.

36. Massenburg BB, Raykar NP, Pawaskar A, et al. Collaboration and innovation in rural surgery. Int Health. 2016;8:367-368.

37. Nepogodiev D, Chapman SJ, Kolias AG, et al. The effect of trainee research collaboratives in the UK. Lancet Gastroenterol Hepatol. 2017;2:247-248. 